\title{
On the influence of fixing the principal point in frame-by-frame multiplanar calibration.
}

\author{
Javier-Flavio Vigueras $^{1} \quad$ Marie-Odile Berger $^{1} \quad$ Gilles Simon $^{2}$ \\ ${ }^{1}$ LORIA - INRIA Lorraine ${ }^{2}$ LORIA-UHP Nancy 1 \\ Campus Scientifique, BP 239, 54506 Vandœuvre-lès-Nancy, FRANCE
}

\begin{abstract}
Calibrating sequences were a camera moves without zooming is often considered as a pure viewpoint estimation problem. Here, we demonstrate that considering varying principal point while keeping the focal length constant allows us to obtain a reprojection error similar to the one obtained with full calibration while imposing existing constraints on the camera (no zooming). Then, we propose a robust calibration process which can be used for any scene that contains planar structures. Robustness is obtained by estimating independently the principal point position and the viewpoint.
\end{abstract}

\section{Introduction}

Camera calibration is an important task in many vision and media applications. For example, when augmenting real video sequences with synthetic $3 \mathrm{D}$ elements, the camera calibration is prerequisite in order that the real and the virtual objects be properly aligned.

In this paper, we consider the common practical case where the camera moves without zooming. This is a very common situation in augmented reality when the user moves with a small camera fixed on a head mounted display. Hence the intrinsic camera parameters should be constant over the sequence. A first contribution of this paper is to prove that considering varying principal points but constant focal length over the sequence leads to more accurate and more stable calibration results.

Our second contribution is to propose an efficient and robust framework for calibrating such a sequence for scenes which contain planar structures. In order to avoid undesirable compensations between intrinsic and extrinsic parameters [1], the principal points and the viewpoints are computed independently for each frame. This can be done thanks to the center line constraint [2]. In addition, we propose a statistical propagation of the detection errors to improve the accuracy of the principal point.
Section 2 illustrates the need of varying the principal point for calibration purpose. Section 3 proposes a framework for calibrating a camera by estimating the principal point in every image. Finally, section 4 shows some experimental results in the context of augmented reality.

\section{Calibration problems}

Camera calibration over a sequence is often considered as either a full calibration process (when all the camera parameters are unknown) or as a viewpoint computation process when the intrinsic parameters are supposed constant and estimated beforehand. In this section we prove that even though camera moves without zooming over a sequence, accurate calibration cannot be obtained with constant intrinsic parameters. Besides, we show that constant focal length and varying camera parameters lead to a reprojection error which is similar to the errors obtained with full calibration, while imposing existing constraints (no zooming is performed).

A video sequence of our calibration target was shot without zooming nor focusing. Let $\mathbf{P}=\mathbf{K}(\mathbf{R} \mid \mathbf{t})$ be the projection matrix recovered from classical calibration techniques, where $\mathbf{R}$ and $\mathbf{t}$ are the orientation and position of the camera (viewpoint), and $\mathbf{K}=\left(\begin{array}{ccc}f & 0 & u_{0} \\ 0 & \tau f & v_{0} \\ 0 & 0 & 1\end{array}\right)$ is the intrinsic parameters matrix. Camera calibration is performed by computing the parameters $\left(f, u_{0}, v_{0}, \mathbf{R}, \mathbf{t}\right)$ that minimize the average reprojection error:

$$
\min _{\left(f, u_{0}, v_{0}, \mathbf{R}, \mathbf{t}\right)} \frac{1}{N} \sum_{k=1}^{N}\left\|\mathbf{u}_{k}-\mathbf{P}\left(\mathbf{x}_{k}\right)\right\|^{2}
$$

given the 3D-2D matches $\left(\mathbf{x}_{\mathbf{k}}, \mathbf{u}_{\mathbf{k}}\right)$. It is assumed that the aspect ratio $(\tau)$ is fixed and that the image axes are orthogonal.

The classical 9-parameters calibration has been performed on each image of the sequence. The reprojection error is plotted as reference in Fig. 1.a. We also performed calibration by fixing the intrinsic parameters. Optimization 


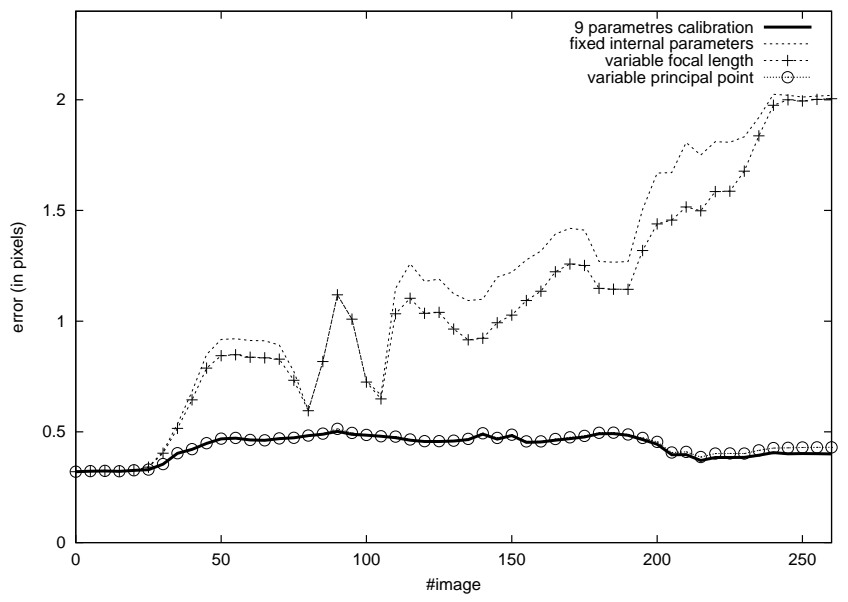

(a)

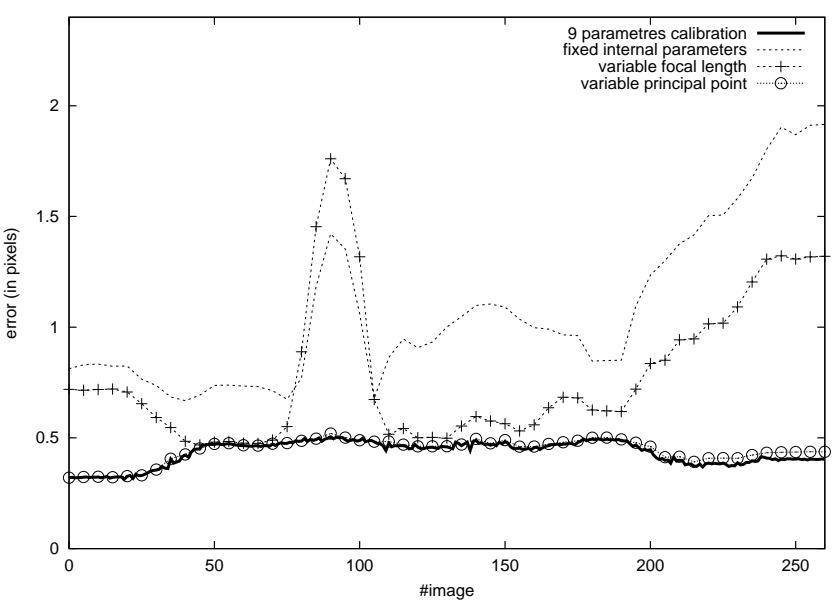

(b)

Figure 1. Comparison tests. (a) Reprojection errors: constant intrinsic parameters are the ones computed from image 0. (b) Reprojection errors: constant intrinsic parameters are the ones computed using a bundle adjustment technique.

was then achieved on the extrinsic camera parameters. Fig. 1.a exhibits the reprojection errors when the internal parameters are fixed to the values computed in frame 0 . Fig. 1.b shows the errors when the internal parameters are computed with a bundle adjustment technique over the set of frames. These plots clearly prove that fixing intrinsic parameters lead to large reprojection errors. The average error is 2 pixels and for some points the error is up to 6 pixels.

These experiments suggest that non constant intrinsic parameters must be used even when no zooming or focusing is performed. In order to know which parameters are more suitable to describe the observed deviations and which ones should remain fixed, we conducted further calibration experiments using:

- a variable focal length and a fixed principal point

- a fixed focal length and a variable principal point

These experiments clearly show that (Fig. 1.a) fixing the principal point also leads to a large residual error. Fortunately, a fixed focal length and a varying principal point gives a reprojection error which is very similar to the one obtained with the 9-parameters calibration.

As the height of the camera was constant during acquisition, the $z$-component of translation in the world coordinates remains constant for all the sequence. The standarddeviation of this parameter for the four cases are:

\begin{tabular}{|c|c|c|}
\hline case & $t_{z}(\mathrm{~mm})$. & $\sigma_{t_{z}}(\mathrm{~mm})$. \\
\hline 9 parameters calibration & 533.76 & 2.97 \\
fixed internal parameters & 540.12 & 5.95 \\
variable focal length & 563.35 & 22.02 \\
variable principal point & 545.50 & 1.85 \\
\hline
\end{tabular}

This table further demonstrates that the stability is better when the principal point varies and the focal length is fixed. To conclude, even if the intrinsics are theoretically constant, calibration is improved by considering constant focal length and a varying principal point. Intuitively, such varying parameters allows to cope with the fact that the pinhole model is only an approximation of a camera. In the following, we describe a robust and accurate calibration framework which implements this idea.

\section{The Calibration framework}

We then propose a calibration process where the set of parameters $\left(u_{0}, v_{0}, \mathbf{R}, \mathbf{t}\right)$ are estimated frame-by-frame. The focal length is constant and is estimated beforehand. This process can be used in any environment which contains some planar structures. The main interest of our framework is to avoid the compensation effects that often appear between intrinsic and extrinsic parameters by estimating independently the principal point position and the extrinsic parameters. The Center Line (CL) constraint [2] is here used to estimate the intrinsic and extrinsic parameters independently. In addition, we propose a robust estimation of the principal point. Then, our framework for camera calibration follows a two-step procedure:

- The principal point is estimated at the current frame by means of the CL constraint applied over several planes.

- The viewpoint is computed using the updated principal point position and the constant focal length from any viewpoint algorithm. 


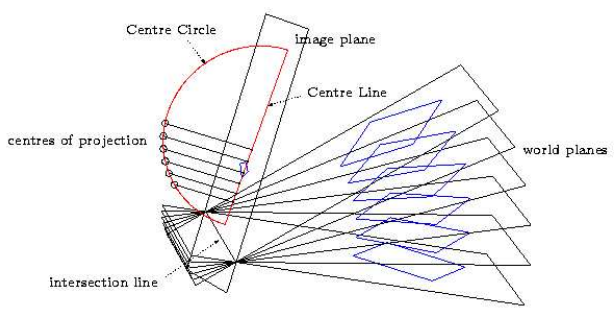

Figure 2. Poncelet's theorem (from [2]).

In the following, we first recall the fundamental center line constraint. Then the calibration algorithm is described.

\subsection{Center Line Constraint}

The Center Line constraint is based on Poncelet's theorem [5] that states:

When a planar figure is the central projection of another planar figure, these figures remain in perspective correspondence when one rotates the plane of the first around its intersection with the plane of the second; the center of projection then lies on a circle, in a plane perpendicular to this intersection. As a result, if one projects this circle orthogonally onto the image plane, one obtains a line segment that is the locus of the principal point (see fig.2).

The equation of the straight line containing the principal point, namely the Center Line, is given by [2]:

$$
A u_{0}+B v_{0}+C=0
$$

where

$$
\begin{aligned}
A= & -\tau^{2}\left(H_{31}^{2}+H_{32}^{2}\right)\left(H_{31} H_{12}-H_{11} H_{32}\right) \\
B= & -\left(H_{31}^{2}+H_{32}^{2}\right)\left(H_{31} H_{22}-H_{21} H_{32}\right) \\
C= & \tau^{2}\left(H_{31} H_{12}-H_{11} H_{32}\right)\left(H_{11} H_{31}+H_{12} H_{32}\right) \\
& +\left(H_{31} H_{22}-H_{21} H_{32}\right)\left(H_{21} H_{31}+H_{22} H_{32}\right)
\end{aligned}
$$

and $H$ is the homography transformation between a plane in the $3 \mathrm{D}$ space to the corresponding 2D image.

\subsection{Principal Point Estimation}

In the seminal work about CL, the authors assumed that the principal point position is constant over a sequence and proposed to determine it as the intersection of the centerlines that correspond to a given plane which is visible in several images. Here, we use this constraint in a different way: the CL constraint is used with several visible planes in every image. As a result the principal point is updated in each image by intersecting the corresponding center lines.

In the original work about CL [2], statistical variations on homography estimation or lines intersection are not considered. However, the estimations are sensitive to some factors as the plane orientation, the number of key-points or their distribution in the image. Then, we suggest to take into account the statistical fluctuations on key-points used to compute homographies, and propagate these deviations up to the principal point computation.

Computing the homographies between two planes is a well known problem that is addressed in [3]. The deviations on the homography coefficients can be estimated as a function of the statistical variations on the key-points that are extracted and matched through the sequence. Given the covariance one the homographies for the $N_{p}$ observed planes, the center lines are obtained by using the Eq. 1, and the propagated covariance is approximated by:

$$
V\left[A_{i}, B_{i}, C_{i}\right]=J_{i} V[H] J_{i}^{T}
$$

where $J_{i}$ is the Jacobian matrix of the line parameters $\left(A_{i}, B_{i}, C_{i}\right)$ respect to the coefficients of $H_{i}$.

A robust estimation of the principal point can now be computed as the intersection of the center lines weighting each distance as a function of the covariance [4]:

$$
\left(u_{0}, v_{0}\right)=\operatorname{argmin} G\left(u_{0}, v_{0}\right)=\sum_{i=1}^{N_{p}} \frac{\left\|\left(A_{i}, B_{i}, C_{i}\right) \cdot P_{c}\right\|^{2}}{P_{c}^{t}\left(V\left[A_{i}, B_{i}, C_{i}\right] P_{c}\right)}
$$

where $P_{c}=\left(u_{0}, v_{0}, 1\right)^{T}$. The main advantage of optimizing this function, instead of the sum of geometric distances to the lines, is that $G$ takes into account that noise does not affect homogeneously the different $\mathrm{CL}$, and it allows us to reduce the sensibility of the principal point to poorly estimated lines (see Fig. 4)

\section{Application to real-time camera tracking}

This framework has been applied to real-time camera tracking for augmented reality. The system described in [6] is used to compute the extrinsic parameters of the camera. This system is based on correspondences of key-points that are located on planar structures.

Results were obtained on a 1000-frames sequence of a room corner. Artificial markers were added into the scene in order to compute the CL constraints (figure 5). One important point is that it is not necessary to know the relative positions of the markers, as each marker provides one CL constraint independently from the others.

Figure 3 shows the evolution of the principal point over the sequence. An example of the estimated principal point is shown in Fig. 4: as marker 1 lies in a nearly frontoparallel plane, the covariance of the corresponding CL is higher than for the other planes. Consequently, the principal point is closer to the lines issued form markers 2 and 3 . The Figure 5 shows the reprojection of the scene in frame 600 , when the principal point is kept constant in the middle of the image (fig 5.b) or is updated using the method 


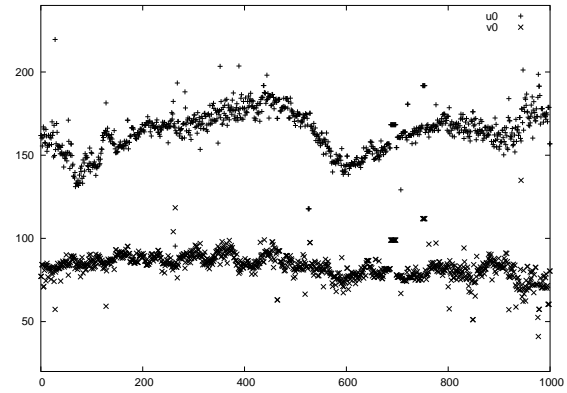

Figure 3. Evolution of the computed principal point over the sequence.

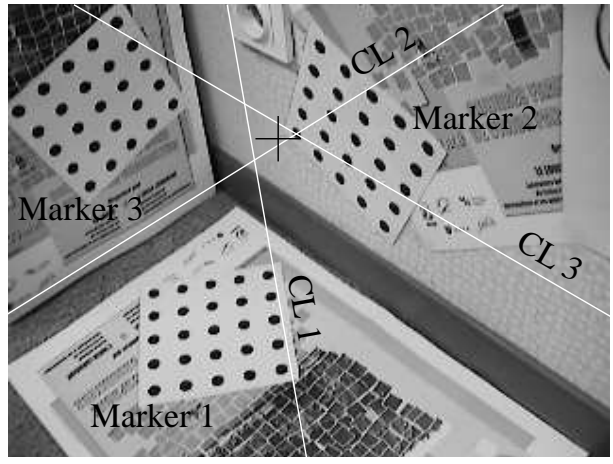

Figure 4. Estimated principal point and center lines for one frame of the sequence.

described in section 3 (fig 5.c). In both cases a drift is observed, due to approximation errors that accumulate over time (this inevitable drift is mentioned in [6]). However, the reader may notice that the drift is much less important when the principal point is updated (see for example the basis of the vertical planes).

\section{Conclusion}

The paper has demonstrated that using a variable principal point and a fixed focal length is a convenient way to describe a moving camera without zooming nor focusing. For scenes containing planar structure, we show that the intrinsic and the extrinsic parameters can be estimated independently, giving rise to a robust calibration scheme.

Acknowledgements This research is being supported with EC-grant IST-2000-28707 (ARIS project).

\section{References}

[1] L. Agapito, E. Hayman, and I. Reid. Self-calibration of rotating and zooming cameras. IJCV, 45(2), Nov. 2001.

[2] P. Gurdjos, A. Crouzil, and R. Payrissat. Another way of looking at plane-based calibration: the centre circle constraint. In ECCV'02, volume IV, pages 252-266, 2002. (a).

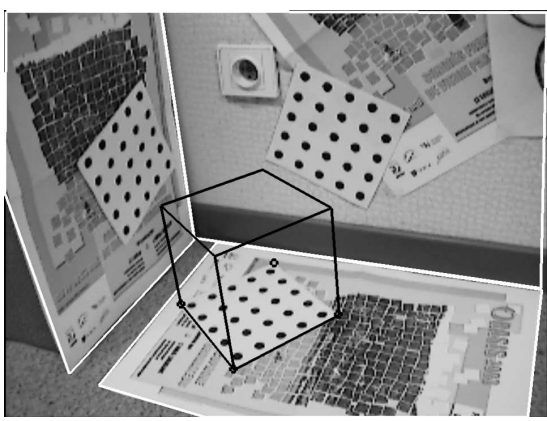

(b).

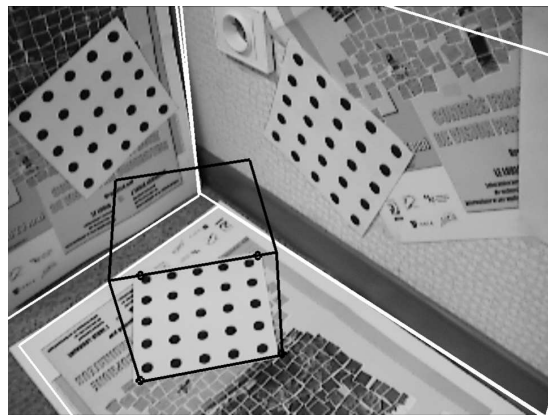

(c).

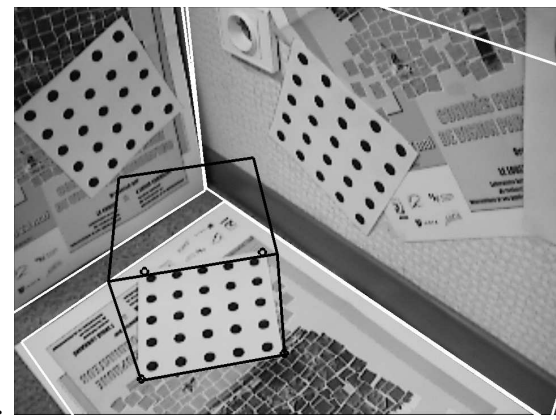

Figure 5. Results obtained on a 1000-frames sequence of a room corner. (a) A multi-planar structure is defined and a cube is added in the first image of the sequence. (b),(c) Reprojection of the scene in frame 600 when the principal point is fixed (b) or is changed frame-by-frame (c).

[3] R. I. Hartley and A. Zisserman. Multiple View Geometry in Computer Vision. Cambridge University Press, ISBN: 0521623049, 2000.

[4] K. Kanatani. Geometric Computation for Machine Vision, volume 37 of Oxford Engineering Science Series. Clarendon Press, 1993.

[5] J.-V. Poncelet. Applications d'analyse et de géométrie - Traité des propriétés projectives des figures. Tome I. Imprimerie de Mallet-Bachelier, Paris, 1862.

[6] G. Simon and M.-O. Berger. Real time registration of known or recovered multi-planar structures: application to augmented reality. In $B M V C^{\prime} 02$, pages 567-576, Sept. 2002. 